Service social

\title{
La maladie mentale comme problème social
}

\section{Henri Dorvil}

Volume 39, numéro 2, 1990

Les problèmes sociaux

URI : https://id.erudit.org/iderudit/706476ar

DOI : https://doi.org/10.7202/706476ar

Aller au sommaire du numéro

\section{Éditeur(s)}

École de service social de l'Université Laval

\section{ISSN}

1708-1734 (numérique)

Découvrir la revue

Citer cet article

Dorvil, H. (1990). La maladie mentale comme problème social. Service social, 39(2), 44-58. https://doi.org/10.7202/706476ar

\section{Résumé de l'article}

L'auteur aborde la maladie mentale comme problème social. Il adopte un point de vue social et s'oppose à une analyse trop exclusivement biologique du phénomène en question. À l'aide de multiples exemples, tant historiques que contemporains, il s'efforce de démontrer que ce sont les normes et les valeurs " qui régissent la construction de l'anormalité dans une collectivité " et qu'en conséquence, la déviance, qu'implique ce type de comportement est bel et bien " un problème social avant d'être une maladie ». Il souligne aussi que la perception et la définition de la déviance évoluent dans le temps et l'espace. Finalement, il situe l'analyse du phénomène de la maladie mentale par rapport aux principales théories explicatives des problèmes sociaux et il rappelle les disparités dans la répartition des troubles mentaux selon les classes sociales. 
Henri Dorvil, professeur au Département de travail social de I'Université du Québec à Montréal. Chercheur au Groupe de recherche sur les aspects sociaux de la prévention (GRASP), Université de Montréal.

\section{La maladie mentale comme problème social}

Henri Dorvil

Le statut de cette forme de perturbation comportementale généralement désignée sous le vocable de maladie mentale a toujours été ambigu. Problème scientifique autant que problème social, elle $a$ été considérée, dès le départ, par les tenants des sciences sociales, au même titre que la délinquance juvénile, le suicide, l'alcoolisme chronique, la déficience intellectuelle, le chômage, la dépendance chronique etc. À l'origine, soit principalement avant la révolution industrielle, ce trouble du comportement n'était pas reconnu comme une maladie; c'était une condition sociale acceptée ou réprimée, semblable à la pauvreté ou à l'itinérance. De nos jours encore, signe indicatif, des psychiatres remettent en question certains aspects pour le moins nébuleux de la sémiologie psychiatrique. Au cours de I'histoire, les malades mentaux ont été successivement pris en charge par divers types de guérisseurs : shamans, hommes de loi, gardiens, religieux, neurologues, psychiatres. Ainsi le traitement moral et le traitement religieux ont précédé l'arsenal neuroleptique ou antipsychotique d'aujourd'hui. De plus, historiquement, les malades mentaux ont partagé les lieux de contrôle social avec les pauvres, les prisonniers, les individus de moralité douteuse.

Dans un premier temps, je vais distinguer maladie mentale et maladie. Ensuite, je présenterai la maladie mentale sous l'angle de la déviance, de la marginalité, avant de l'aborder en tant que problème social. La maladie mentale fait de plus en plus l'objet de brillantes spéculations neurobiologiques et cette forme de psychiatrie semble voguer allègrement vers $d^{\prime}$ autres sommets. Cet article s'inscrit à contre-courant et veut renouer avec les thèses de l'étiologie sociale de la maladie mentale. 


\section{Maladie mentale et maladie}

Les théories et les pratiques en sciences sociales dans le domaine de la santé se basent sur la maladie diagnostiquée. Les chercheurs et les praticiens de ce champ ne remettent pas habituellement en question l'objectivité scientifique de l'infarctus du myocarde, de tel type de cancer, de l'hypertension ou du sida. À partir de là, considérant la maladie comme phénomène social' au moins autant que phénomène biologique ou psychologique, ils apportent divers types d'explications. Par exemple, depuis toujours l'organisation de la société est cause de maladies, particulièrement pour les classes les plus défavorisées; l'espérance de vie est liée à la pauvreté, à la sousculture et à l'inégalité des chances.

Les choses se passent différemment en psychiatrie où la notion même de maladie diagnostiquée est contestée, non seulement par les tenants des sciences sociales mais par les psychiatres eux-mêmes. Qu'il suffise de rappeler brièvement la divergence de vue des psychiatres concernant le diagnostic des mêmes symptômes, le passage arbitraire d'une entité à l'autre, le pronostic de la dangerosité ${ }^{2}$. On se souviendra de la déclassification ex cathedra de I'homosexualité ${ }^{3}$ comme maladie mentale par l'association psychiatrique américaine en 1974. Et que dire des diagnostics erronés menant à des gâchis médicamenteux ? Des psychiatres et des chercheurs en neurobiologie ${ }^{4}$, sensibilisés à ce problème, nous mettent en garde :

Les délires paranoïdes et les hallucinations ne sont pas rares dans la manie ou la dépression. Le tableau paranoïde entraîne chez le clinicien non averti un diagnostic de schizophrénie paranoïde ou de réaction schizo-affective. Les patients victimes de ces diagnostics erronés sont astreints à des cures de neuroleptiques. Or, on sait qu'il y a une incidence élevée de dyskinésie tardive chez les maniacodépressifs dont le traitement antipsychotique a duré plus d'un an. Des études au Centre Hospitalier Louis-H. Lafontaine chez les maniaco-dépressifs traités aux neuroleptiques ont montré une incidence de dyskinésie tardive variant de $74 \%$ à $86 \%$. Ces résultats ont été confirmés depuis par plusieurs études américaines.

Henri Collomb ${ }^{5}$, un médecin au-dessus de tout soupçon antipsychiatrique, écrit sereinement : " [...] dans la plupart des cas, il (" le fou ") n'est pas malade au sens médical du terme. Les examens biologiques seront tous négatifs ». Quant à Szasz', un autre psychiatre dans ce concert de voix contre le traitement médical de la maladie mentale à l'image des autres maladies, affirme (nous traduisons) :

Je postule (I submit) que la maladie mentale est un mythe. Les corps sont des objets physiques : les esprits (minds), quoiqu'ils puissent 
être, ne sont pas des objets physiques. Par voie de conséquence, des maladies mentales (telles que la dépression nerveuse ou la schizophrénie) ne sauraient exister dans le sens où les maladies du corps (telles que les os cassés ou les peaux ulcérées) elles, existent.

Ce psychiatre clame assez fort que la maladie mentale n'a pas de base organique et ce, même pour la schizophrénie. Ce n'est que par analogie qu'on peut parler de maladie mentale comme maladie de l'âme, maladie de l'esprit, comme autrefois I'on appelait les prêtres des médecins de l'âme. Mais, note Szasz, depuis le Moyen Âge environ, la société a tendance à étendre la notion de maladie à toute une panoplie de malheurs existentiels qui peuvent accabler I'homme : malheurs économiques, moraux, raciaux, politiques ${ }^{7}$ et, pouvons-nous ajouter, la maladie mentale constituant la « pathologie " où la composante morale et normative est la plus évidente; c'est par le biais de la filière psychiatrique que la médecine a envahi tout un champ de problèmes sociaux : homosexualité, alcoolisme, suicide, naissance, stress, vieillissement, etc.

Un autre facteur différencie la maladie mentale de la maladie physique, il s'agit de la relation avec autrui, qui constitue la cause principale du problème de la maladie mentale. Or, on fait peu de cas de cette dimension. Ce rapport avec autrui se trouve vite brouillé par l'étiquette "diagnostic", les médicaments, le lit d'hôpital, les rechutes, I'assistance sociale. L'individu-sujet devient vite objetmaladie. Le professeur Collomb ${ }^{8}$ dit justement :

Réduire la folie à la maladie, c'est supprimer la folie en tant qu'objet de scandale. C'est éviter de reconnaître à la folie sa dimension humaine, ce qui la rendrait insupportable. C'est aussi mettre entre I'homme normal et le malade mental la science médicale, écran qui évite le face à face intolérable. La folie questionne douloureusement I'homme dit normal, soumis aux contraintes sociales. Elle est émergence d'une autre vérité plus réelle que la réalité reconnue par les autres, imposée par l'ordre social. Elle est aussi liberté, liberté absolue qui refuse l'ordre social.

On comprend dès lors que la folie fascine tout en faisant peur. De là, la nécessité pour l'individu et la société de se protéger contre ce danger pouvant mettre en péril l'un et l'autre.

Contrairement à la maladie physique où les signes cliniques sont en général évidents, il existe parfois une confusion totale entre l'état de maladie mentale et l'état de santé mentale. C'est le caractère erratique du discours et du comportement qui peut départager l'état anormal de l'état normal et non les examens cliniques habituels : auscultation stéthoscopique, prise de sang, de tension, test d'urine, électrocardiogramme, biopsie, radiographie des poumons, etc. La différence s'avère très grande avec l'orthopédie par exemple, où l'on 
n'hospitalise pas quelqu'un s'il n'a pas un membre fêlé ou cassé. La maladie mentale n'est pas structurée à partir de symptômes clairs et objectifs de manière à permettre un diagnostic univoque. De plus, alors qu'en médecine physique le médecin se base sur la foi des symptômes rapportés par le patient pour bâtir son diagnostic, en psychiatrie la parole du fou est suspecte et non avenue ${ }^{9}$.

Par ailleurs, si la maladie mentale ne renvoie pas toujours à une anormalité biologique, elle ne renvoie pas non plus au rôle social d'être malade, idéalement défini par Parsons ${ }^{10}$. Contrairement aux autres malades, le malade mental ne se conforme pas généralement aux divers éléments qui composent ce " rôle de malade ", c'est-à-dire recours, collaboration, soumission au médecin, effort pour se rétablir afin de réintégrer ses rôles sociaux. Quand quelqu'un souffre de gastro-entérite, il sollicite de lui-même l'intervention médicale. Tel n'est pas le cas pour la maladie mentale où l'hospitalisation (du moins la première) se fait sous la contrainte, dans la plupart des cas : la famille, le milieu de travail, le corps judiciaire obligent l'individu bizarre à voir le psychiatre. De plus, si la déclaration d'une maladie physique concourt à soustraire le malade à ses obligations habituelles, il n'est guère recommandé de soustraire le patient psychiatrique à ses tâches coutumières ${ }^{11}$. Au contraire, l'occupation est jugée thérapeutique, le travail considéré comme la voie royale de la réhabilitation, tout d'abord pour se reconnecter avec la réalité en ne laissant pas prise au délire, ensuite pour réintégrer son image et son schéma corporels et sa relation aux objets.

Il existe d'autres différences entre maladie mentale et maladie physique. Même si elle suscite des réactions négatives, la maladie mentale n'est pas contagieuse comme le sida, ne tue pas comme le cancer ou l'infarctus du myocarde. De plus, si la médecine arrive à contrôler l'agitation et la fureur de la folie, les annales médicales n'ont guère enregistré à ce jour de guérison éclatante comme dans le cas des maladies contagieuses.

À côté des grandes catégories diagnostiques que sont la névrose et la psychose, la nosographie populaire retient d'un côté les fous tranquilles, de l'autre, les fous furieux. Les premiers ressemblent aux autres malades : dociles, obéissants, ils collaborent au traitement et se conforment habituellement au "rôle de malade". Les seconds sont récalcitrants et se trouvent plus ostracisés que les fous tranquilles. Une catégorie attire la pitié, l'autre inspire la peur, le rejet, un peu comme les "véritables pauvres " d'autrefois, par opposition aux " mauvais pauvres " aptes au travail mais paresseux, voire dangereux. De plus, toutes catégories confondues, il y a dans la maladie mentale cet aspect d'irresponsabilité, d'incapacité, d'imprévisibilité, de violence, que l'on ne retrouve guère dans les maladies physiques. La 
société protège le fou et se protège aussi contre la folie, attitude qu'elle adopte uniquement en temps d'épidémie, lors de maladies contagieuses.

À tout handicap, à toute maladie déclarée est accolé un stigmate. Dans le cas de la maladie mentale, ce stigmate s'avère plus lourd pour les pauvres ayant un accès restreint à la thérapie et ayant peu développé l'estime de soi. Par contre, des schizophrènes ingénieurs, médecins, hommes d'affaires ne sont pas marginalisés, à cause du pouvoir (matériel, symbolique) qu'ils possèdent. Cette différenciation de classe est moins marquée dans le vécu des autres maladies.

Dans certaines cultures, les délires paranoïdes ne sont pas étrangers au tableau clinique de la manie ou de la dépression alors que dans d'autres, le phénomène est plutôt rare, insolite. Ainsi, durant un quart de siècle, des diagnostics de schizophrénie paranoïde ou de réaction schizo-affective ont été portés à l'endroit de ressortissants d'un groupe ethnique bien identifié à Montréal. Or, quoi de plus logique que de retrouver des traces d'idées de persécution dans la dépression d'un malade originaire d'un pays à dictature où règne en plus la sorcellerie ? Il en est de même des pauvres à qui l'on accole plus souvent le diagnostic sévère de schizophrénie ${ }^{12}$. Dans les autres spécialités de la médecine, les critères de diagnostic résistent mieux aux lignes de démarcation de classe et de culture.

Avant de clore cette section, il est utile d'apporter quelques précisions sur l'aspect chronique de la « maladie » mentale. Il faudrait une étude complète pour comparer d'une part la maladie physique chronique et la "maladie » mentale chronique et, d'autre part, la maladie physique aiguë et la " maladie " mentale aiguë, mais tel n'est pas le but de cet article. La récidive est l'essence même de la "maladie» mentale. Pour rejoindre la pensée de Lanteri-Laura ${ }^{13}$, disons que la pathologie mentale s'avère par excellence une pathologie chronique et que, bien plus que dans d'autres spécialités, les affections en cause ont une propension pour ainsi dire naturelle à durer très longtemps. Cet état de fait se reflète aussi dans les écrits. Après une analyse exhaustive des manuels européens et américains de psychiatrie, cet auteur conclut que la chronicité occupe la plus grande place dans une proportion de 8 contre 1 (pathologie aiguë). Cependant, comme le souligne l'auteur, certaines conditions socioéconomiques sont à la base de cette chronicité et déterminent la naissance des asiles. Ceux-ci ne peuvent fonctionner que si la maind'œuvre ne fait pas défaut, et ils ne sont rentables qu'à la condition que les malades y restent assez longtemps pour fournir cette maind'œuvre à bon marché. C'est cette chronicité en puissance de la «maladie " mentale qui, avec le besoin de contrôle social, justifie économiquement encore l'installation d'asiles (mais aussi de prisons 
et de bases militaires) en pleine zone défavorisée et sous-peuplée, alors que la création d'hôpitaux généraux répond à des critères épidémiologiques et démographiques vérifiables.

C'est l'asile qui, en coupant le sujet de la réalité sociale pour plusieurs années et souvent pour toujours, lui fait perdre ses habiletés indispensables à toute vie en société. Aucun hôpital n'agit ainsi et sur une si longue période à l'égard d'un malade. Aucune maladie $n$ 'exige un appareillage aussi complexe de réinsertion sociale que la «maladie " mentale.

Toute cette démonstration sert à illustrer le fait que la " maladie " mentale n'est pas une maladie comme les autres, que tout au moins c'est une entité frontière chevauchant à la fois la maladie et la déviance.

\section{Maladie mentale, déviance, marginalité}

Maladie pour la gente médicale, déviance, marginalité pour les gens du social, la "maladie " mentale n'a pas fini de s'interroger sur son identité. La déviance se définit par l'écart à la norme. Sociologiquement, la déviance est le recours à des modèles de conduite qui se situent à la marge de ce qui est permis. Ainsi conçue, la déviance se trouve en rupture de ban avec le fondement même de toute société qui vise l'ordre social dit naturel. En quoi cette notion peut-elle nous éclairer sur la " maladie » mentale ? Quel en est le lien de parenté ?

Dès le départ il est vrai, la " maladie » mentale a été étroitement associée à cette définition de la déviance. D'entrée de jeu, je peux affirmer que la maladie mentale est déviance sociale avant d'être reconnue comme maladie par la médecine. En effet, le processus de catalogage et de diagnostic psychiatriques ne commence pas au bureau du psychiatre mais au sein de la communauté, souligne Scheper-Hughes ${ }^{14}$. Avant même de se présenter, volontairement ou non, à une consultation psychiatrique, le patient possède déjà une longue et complexe histoire de négociation avec sa famille, ses collègues de travail et ses voisins, quant à la signification de sa conduite déviante. Ces tensions mettent en évidence le rôle primordial de la famille, du voisinage et de la société dans l'évaluation de l'acte du présumé malade mental et dans la reconnaissance des symptômes de la folie. Donc, il existe des normes et des valeurs qui régissent la construction de l'anormalité dans une collectivité. Un acte relevant de la "maladie» mentale est un acte qui transgresse la norme acceptée dans une société, un acte qui fait problème, un acte que ne poserait pas ou ne devrait pas poser un individu appartenant à la dite culture. Bref, c'est ce qu'Ellen Corin ${ }^{15}$ appelle la construction 
culturelle de la maladie mentale. Plusieurs ouvrages consacrés spécifiquement à la "maladie " mentale relèvent de diverses manières cette collision entre la conduite du " malade " mental et les normes de la société ambiante.

Dans une œuvre magistrale, Michel Foucault ${ }^{16}$ établit vers 1656 la date de mutation des normes bourgeoises qui allait forcer ce genre de déviants à rentrer dans le rang ou à se réfugier dans les loges des hôpitaux généraux. Une norme étant totalitaire en soi, le comportement du fou ne devait en rien entrer en compétition avec les normes relatives à l'éthique du travail, à la responsabilité, la capacité, la propreté, l'utilisation rentable du temps, la fixation à un lieu déterminé (adresse), l'individualisme du nouvel Ordre bourgeois. Ce sont les officiers du roi, les gardiens de la paix qui jugeaient de la normalité du comportement sur le plan économique et moral. II n'était guère question alors de critères de "maladie " mentale, mais l'évolution économique et sociale de l'Europe au XVII et au $\mathrm{XVIII}$ e siècle commandait l'institutionnalisation du fou au même titre que toute une kyrielle de déviants avec lesquels on lui reconnaissait un degré de parenté : vagabonds, libertins, prostituées, criminels, etc. Les productifs ne doivent plus être dérangés par les improductifs désormais réformés selon les canons de la morale bourgeoise. Le fou se trouve ainsi banni du circuit des rapports sociaux, à l'instar de tous les individus porteurs, potentiellement ou de fait, de désordre, de violence, de bestialité, de criminalité. C'est cette idée de menace à la dignité humaine, de déchéance, cette peur qui expliquent les mauvais traitements infligés autrefois au fou et son enfermement à l'asile, attaché au mur par le cou ou les pieds ${ }^{17}$. Le fou est frappé de perversité morale avant d'entrer dans la nosologie médicale. Cette déviance constitue bel et bien un problème philosophique et social avant d'être une " maladie ».

Comme on peut le constater, c'est le comportement dérangé et dérangeant qui indique la " maladie " mentale chez un individu, et non un test d'urine ou une prise de sang. André Cellar ${ }^{18}$ qui, dans une thèse de doctorat, examine entre autres le comportement des Amérindiens à l'égard de leurs fous, aboutit à peu près à la même conclusion. La communauté cesse d'être compatissante pour devenir très sévère quand le fou est furieux, agité et qu'il représente un élément de danger pour l'équilibre de la collectivité. Le malaise constitué par la perturbation discursive et comportementale du fou fait alors problème à l'organisation sociale.

Dans un livre récent, j'ai décrit les écarts de langage et de comportement qui distinguent le "malade " mental de l'homme présumément sain ${ }^{19}$. Contrairement au bien-portant, le "malade » 
mental aurait enfreint plus fréquemment le code moral de la municipalité en ce qui a trait à la décence du discours, au respect de la propriété privée, à la capacité civile, au contrôle de sa personne, à l'éthique du travail, ou pour s'être livré à l'errance, à l'alcoolisme, aux veilles de nuit, à l'exhibitionnisme sexuel, au " parler tout seul ». À quoi reconnaissez-vous un "malade " mental, ai-je demandé à mes interlocuteurs ? À sa tranquillité, à l'apparence vestimentaire, au caractère brusque de ses gestes, au fait d'être lunatique, à l'incohérence du discours, répondent-ils. Comme on peut le constater, il n'y a rien de spécifiquement organique dans ces signes de reconnaissance de la " maladie » mentale. II s'agit là de comportements qui peuvent menacer le code éthique et l'organisation de cette collectivité ou tout au moins outrepassent les limites de la bienséance qui est le garant de bonnes relations entre individus.

La ville de Gheel, en Belgique, est connue dans le monde entier pour ses soins aux handicapés mentaux désinstitutionnalisés en milieu rural. Roosens ${ }^{20}$ écrit à ce propos :

L'image de la rue et de la vie en public est d'une banalité consternante. Les pensionnaires se confondent avec les passants. Et si quelque chose nous frappe dans l'expression de leur visage ou la pauvreté de leur vêtement, ils ne se livrent à aucune manifestation extraordinaire, ni ne témoignent d'aucune agitation. Au grand maximum leur allure paraît un peu particulière, insolite. Tous les gens de Gheel connaissent des histoires de patients que des étrangers ont pris pour des habitants normaux, tandis qu'inversement des habitants de Gheel, en parfaite santé, étaient confondus avec des patients. En semaine, et même durant la majeure partie du week-end, le cas du patient qui frappe l'attention du passant se présente peu fréquemment.

En médecine générale, les signes visibles et objectifs démarquant le bien-portant du malade sont plus évidents. Ici ce sont les signes manifestes de la déviance marginalisant les individus étiquetés « malade » mental qui prédominent.

Dans un village du Bourbonnais, Ainay-Le-Château, des fous partagent l'existence quotidienne d'une population dotée de toutes ses facultés mentales. Denise Jodelet ${ }^{21}$, qui fait l'ethnologie de cette psychiatrie communautaire, constate que les villageois qui hébergent aujourd'hui des pensionnaires ont été élevés dans cette proximité de la maladie mentale. D'où une sorte de banalisation de l'étrangeté, qui s'accompagne d'une interrogation permanente sur les frontières fragiles séparant la santé mentale de la maladie, et de l'impression obsédante que nul n'est assuré de ne jamais passer de "l'autre côté ", c'est-à-dire du côté du monde de la folie. L'individu serait sain par sursis. 
Il faut ajouter à l'argumentation en disant que la déviance, ou du moins son évaluation, change avec le temps, selon l'évolution des mentalités. Ainsi jusqu'à la fin du Moyen Âge, l'Occident avait toujours accordé I'hospitalité à la folie. II en a fait une "maladie " par la suite. La déviance se présente différemment suivant les lieux. Ce qui est déviant ailleurs ne l'est pas forcément ici. Des comportements qui n'étonnent personne en Inde, écrit Roland Jaccard ${ }^{22}$, qui sont même considérés comme l'expression d'une grande sagesse, seraient stigmatisés, d'après nos critères occidentaux, carrément schizophréniques ou délirants. La maladie en général ne varie pas beaucoup en fonction de ces deux facteurs. Un cancer de l'œsophage en Inde répond aux mêmes critères objectifs que ceux qui sont retenus en Amérique.

Mais cet écart à la norme que suppute la définition de la déviance peut être appréhendé différemment. Le concept de marginalité par exemple amène plus de dynamisme pour aborder le stigma qui auréole négativement le déviant. Le stigma, rappelons-le, est plus fort sur la " maladie " mentale que sur la très grande majorité des autres maladies et hypothèque la carrière de l'individu qui en est atteint. Les théories sur la marginalité éclairent davantage les différences que la " maladie » mentale accuse à l'égard des autres maladies, et mettent plus en évidence encore ses affinités avec la notion de problème social que nous aborderons dans la prochaine section. Selon I'historien français Roger Chartier ${ }^{23}$, il existe trois types de marginalité : la marginalité comme acte volontaire, par déclassement économique ou comme produit du regard social. Tout d'abord, on peut se mettre soi-même en marge de la vie sociale : tout un courant de spiritualité médiévale a pu conduire à cette attitude. Les Hippies des années 1960 et les moines héritent de cette tradition par leur attitude de retrait. On peut parler ensuite de marginalité par déclassement économique, c'est-à-dire par l'exclusion des processus de production. Par exemple, l'affaissement de l'économie du début des années 1980 a donné lieu à cette réorganisation du marché du travail qui s'exprime depuis en une fragmentation de l'emploi (temps partiel, poste temporaire, sous-traitance, etc.), en des fermetures d'usines, et exige plus de compétences à cause de l'apparition de nouvelles technologies. Une précarisation de l'emploi peut déclasser une population (femmes, travailleurs non spécialisés, etc.), voire la clochardiser. En dernier lieu, la marginalité peut provenir de la mouvance du regard social : les marginaux seraient alors ceux qu'une société donnée désigne comme tels par ses dominants (Église, milieux de notables, etc.) par des discours explicites ou par des pratiques sociales. En bref, la marginalité résulte de la confrontation entre les transformations de la condition socio-économique du plus grand 
nombre et les représentations des marginaux dans l'imaginaire des dominants. À un moment donné, les images de telle catégorie sociale ("malades» mentaux, itinérants, etc.) deviennent prégnantes et enclenchent le processus d'exclusion. La nouvelle sensibilité du dominant produit la haute visibilité du comportement non désirable. Les deux derniers types de marginalité recoupent de part en part I'histoire de la mouvance des attitudes en Occident à l'égard de la folie. Comme le soutient Becker ${ }^{24}$, la déviance $n^{\prime}$ est pas une qualité inhérente au comportement, mais le résultat de l'interaction entre la personne qui pose l'acte et ceux qui y réagissent. La relation avec autrui n'est-elle pas le problème fondamental de la maladie mentale comme le soutenait plus haut le psychiatre Henri Collomb ${ }^{25}$ ?

\section{Maladie mentale, problème social}

Cette troisième section est consacrée à la " maladie mentale ", plus spécifiquement conçue comme problème social :

Un problème social ${ }^{26}$ se définit comme une situation touchant un nombre significatif de personnes et qui est considéré par elles ou par une portion significative de la société comme une source de difficulté ou de désagrément, susceptible d'être améliorée.

Wirth $^{27}$ va un peu dans le même sens. Pour lui, il y a problème social quand une situation existante diverge d'une autre situation qui est préférée par la majorité parce qu'elle est en accord avec certaines valeurs d'une société donnée. Un problème social existe alors seulement quand certaines personnes considèrent une situation existante comme un problème, que ces personnes soient des acteurs ou des spectateurs.

Derrière ces définitions se profilent deux corps théoriques : la théorie de la désorganisation sociale et celle ayant trait aux conflits d'intérêts et de valeurs. Selon la première théorie, les problèmes sociaux apparaissent à partir de situations dans lesquelles les individus sont tellement en rupture avec leur culture qu'ils sont incapables de comprendre suffisamment autrui pour entrer en relation avec lui. Cela va sans dire que le concept d'anomie de Durkheim dans son étude sur Le suicide ${ }^{28}, n^{\prime}$ est pas étranger à cette théorie. Nous vient à l'esprit l'étude de Znaniecki sur l'intégration des immigrants polonais en Europe et en Amérique ${ }^{29}$, ainsi que celle de Faris (1948) qui analyse les problèmes sociaux sous l'angle de la désorganisation sociale ${ }^{30}$. Au compte de cette même théorie, s'inscrit le Cultural Lag de Herman ${ }^{31}$, pour qui il y a problème social quand une société crée une dynamique de changement sans prévoir de mécanismes pour contrôler un éventuel dérapage. Le problème social réside 
dans l'incapacité de la société à contrôler la logistique du changement social. Selon la deuxième théorie, les problèmes sociaux ne dérivent pas de l'inhabileté des gens à communiquer avec autrui ou à entreprendre des actions collectives, mais d'une opposition fondamentale et rationnelle à propos de valeurs et d'intérêts différents.

Ces deux corpus théoriques qui président au fondement de la notion de problème social doivent nous renseigner aussi sur la " maladie » mentale. On peut résumer les théories en quelques mots clés : rupture avec la culture, anomie d'un côté et divergence d'intérêts et de valeurs de l'autre. La maladie consiste en quelque sorte en une baisse de la capacité homéostatique d'une culture; cette maladie se situe en diffraction par rapport à la culture ambiante. En fin de compte, ce n'est pas tant la façon dont un homme agit qui constitue le problème social, mais la façon dont la majorité le juge par rapport aux normes dominantes. Le fait d'enfreindre une règle remet en cause la culture et compromet l'organisation sociale. C'est la culture qui définit une perturbation discursive/comportementale comme devant relever de la médecine des insensés. Cette stigmatisation s'avère d'une importance primordiale, et pour l'individu étiqueté, et pour la survie de la loi et l'ordre dans toute société. C'est le maintien ou le changement des institutions sociales, représentantes de l'ordre, qui règle l'anomie ou la divergence d'intérêts et de valeurs à l'origine du problème social. Horton ${ }^{32}$ a su situer les enjeux des théories explicatives des problèmes sociaux. Ces théories sont d'ordre normatif puisqu'elles définissent et expliquent les comportements déviants à partir de valeurs fixées socialement ${ }^{33}$. Selon lui, les théories sociologiques modernes de la déviance sont le plus souvent des adaptations de deux modèles fondamentaux qui tirent leur origine de l'histoire et de la pensée sociale du XIXe siècle : le modèle d'ordre et le modèle du conflit. Le premier s'articule autour de la notion d'anomie pour expliquer les conflits et les problèmes sociaux, notion qui suppose "l'ajustement » de cette déviation sociale. Par ailleurs, le modèle conflictuel utilise la notion d'aliénation comme concept explicatif des problèmes sociaux et de la croissance de la déviance dans les sociétés modernes.

Dans un travail antérieur, j'ai été amené à l'utilisation de ce modèle binaire ${ }^{34}$. À mon avis, les interprétations de "l'ordre " de la réalité sociale sont teintées d'un but normatif en ce sens qu'elles cautionnent et maintiennent les valeurs de l'ordre établi dans tel système social donné. II en est de même des interprétations conflictuelles qui prônent de nouvelles valeurs en vue de les faire légitimer, valeurs qui entrent à tout coup en opposition avec celles déjà existantes. En effet, dans un cas comme dans l'autre, on doit constater que la démarche n'est ni neutre, ni descriptive et qu'il existe 
des deux côtés un principe d'action humaine dépendante de telles valeurs sociales données.

Maintenant, si l'on envisage la "maladie " mentale comme problème social selon l'approche de l'ordre, elle s'esquisse à l'intérieur d'une société vue comme un système d'actions unifié par une culture commune. L'entente s'avère unanime à travers les différentes composantes du système : valeurs, modes de communication, organisations politiques. Par le biais de la socialisation, le système social s'attend à une dévotion de tous à son égard. À ce niveau, le comportement anormal du " malade " mental, opposé à la conformité sociale, signifie pour lui l'incapacité de remplir ses rôles sociaux. Il s'agit d'une déficience, voire d'un échec du processus de socialisation. Bien des objectifs s'avèrent hors d'atteinte pour le "malade " mental, ce qui entraîne un certain détachement par rapport au système normatif. Le problème social que pose le " malade » mental en est un de socialisation. Il s'agit d'un échec de la société qui n'a pas réussi à lui inculquer le respect des obligations et des interdits sans quoi la vie collective serait impossible. Le psychisme du «malade » mental ne lui fournit pas les motivations dont il a besoin pour agir et réagir en société. Il est évident que cette approche refuse de remettre en question l'ordre existant et qu'elle impute le manque à l'individu déviant.

Le modèle du conflit se trouve à l'opposé du modèle de l'ordre. Pour les théoriciens du modèle conflictuel, I'harmonie sociale prônée par l'ordre n'est qu'une stratégie de la classe dominante, qu'une rationalisation du mécanisme de contrôle social. Dans cette optique, on ne parlera guère de l'échec du " malade " mental dans l'accomplissement de ses rôles sociaux, mais plutôt de l'incapacité du système dans son état actuel à rencontrer les besoins du plus grand nombre. II y a bien un lien entre cette manière de voir et l'antipsychiatrie qui considère le refus du psychotique à s'engager dans la vie sociale comme une protestation contre les relations sociales existantes, contre le standard du normal. L'aliénation du " malade " mental se manifeste dans le fait qu'il obéit contre son gré à des conventions sociales auxquelles il ne croit pas, ce qui le sépare de sa nature créative. Celui qu'on dit malade recule ${ }^{35}$ devant la destruction de soi que représente l'accès à la normalité. Il s'engage dans une direction opposée, laquelle est toute proche de l'état de véritable santé psychique et de liberté intérieure et interpersonnelle qu'il atteindrait $\mathrm{s}^{\prime} \mathrm{il}$ arrivait à dépasser l'étreinte contraignante et réductrice de la prétendue normalité.

La codification médico-psychiatrique officielle de l'étrangeté du fou date du XVIII siècle, époque d'institutionnalisation massive, d'augmentation d'individus dits lunatiques. C'est l'ère de la recon- 
naissance du pouvoir judiciaire du médecin, de son diagnostic et de son traitement. Il s'agit là d'une opération importante mais de second ordre : passation de pouvoir d'une élite à l'autre, changement de garde au poste de vigie des normes. La première caractéristique stigmatisante de la folie (refus, marginalisation, mise à l'écart) a été décidée par la société bien longtemps avant. Ce serait une erreur à la fois historique et épistémologique que de l'oublier. La " maladie " mentale a été fixée et reconnue à partir de mutations de société, de conditions d'organisation essentiellement politiques qui fluctuent avec le temps. L'installation de nouvelles valeurs commandait la disparition du fou des circuits sociaux parce qu'il est non compatible avec les paramètres économiques, moraux du nouvel Ordre. Tout changement dans la société (récession économique, chômage, transformation de la structure familiale, resserrement des normes, retrait de l'État des programmes de protection sociale, etc.) a toujours eu pour effet de faire basculer dans la folie un nombre de plus en plus grand d'individus.

Aujourd'hui comme hier, il y a disparité de classes sociales dans la répartition des troubles mentaux. De par ses conditions de vie, la classe défavorisée est démunie de moyens préventifs, surtout en période de crise. S'agit-il de "maladie " mentale déclarée, cette classe pauvre s'en sort plus difficilement, ne disposant pas de réserve de biens matériels et ne participant ni à la définition des normes sociétales, ni au contrôle social. Contrairement à la classe aisée, elle ne jouit pas de l'estime de soi à même de renforcer l'immunité contre les troubles mentaux. On ne saurait oublier cette considération, même dans l'engouement des brillantes spéculations dopaminergiques $^{36}$ de l'heure.

\section{Notes et références}

1 M. Renaud (1985). "De la sociologie médicale à la sociologie de la santé : Trente ans de recherche sur le malade et la maladie " : 281-291, dans Traité d'anthropologie médicale, ouvrage collectif sous la direction de J. Dufresne, F. Dumont et Y. Martin, Québec et Lyon : Presses de I'Université du Québec; IQRC et Presses Universitaires de Lyon.

${ }^{2}$ J. Poupart et al. (1982). "L'expertise de la dangerosité ", Criminologie, vol. $\mathrm{XV}, \mathrm{n}^{\circ} 2$.

${ }^{3}$ P. Thuillier (1989). "L'homosexualité devant la psychiatrie ", La recherche, vol. $20, \mathrm{n}^{\circ} 213$.

${ }^{4}$ P. Desrosiers, L. Turnier, J. Hillel et G. Chouinard (1990). "Le diagnostic bipolaire ", Le médecin du Québec, vol. 25, n 8, août $1990: 77$.

${ }^{5}$ H. Collomb (1980). "Editorial Comment", Social Science and Medecine, vol. 14B. 
${ }^{6}$ Cité dans M.R. Kaufman (1967). "Psychiatry: Why medical or social model ? ", Archives of general psychiatry, vol. $17:$ 347-360.

7 T.S. Szasz (1980). Cité dans Transitions, $\mathrm{n}^{\circ} 3$.

${ }^{8} \mathrm{H}$. Collomb, op. cit. : 81-84.

${ }^{9} \mathrm{H}$. Dorvil (1985). "Types de sociétés et de représentations du normal et du pathologique : la maladie physique, la maladie mentale " : 305-332, dans Traité d'anthropologie médicale, op. cit.

${ }^{10} \mathrm{~T}$. Parsons (1970). "Structure sociale et processus dynamique : Le cas de la pratique médicale moderne", dans C. Herzlich, Médecine, maladie et société. Paris et La Haye : Mouton.

$"$ Voir par exemple K.T. Erikson (1957), "Patient Role and Social Uncertainty : A Dilemma of the mentally ill ", Psychiatry, vol. 20; R. Sobel et A. Ingalls (1964), "Resistance to Treatment : Explorations of the patient's sick role ", American Journal of Psychotherapy, vol. 18; N.K. Denzin et S.S. Spitzer (1966), "Path to mental hospitals and staff predictions of patient role behaviour", Journal of Health of human behavior, vol. 7; C.L. Peterson (1986), "Changing Community attitudes toward the chronic mentally ill through a psychological program ", Hospital and Community Psychiatry, vol. $37, \mathrm{n}^{\circ} 2$.

${ }^{12}$ A.B. Hollingshead et J.C. Redlich (1958). Social Class and Mental IIIness. New York : John Wiley \& Sons.

${ }^{13} \mathrm{G}$. Lanteri-Laura (1972). " La chronicité dans la psychiatrie française moderne - note d'histoire théorique et sociale ", Annales, vol. 27, n' 3 .

${ }^{14} \mathrm{~N}$. Scheper-Hughes (1987)."Mental in Southie : individual, family and community responses to psychosis in South Boston ",Culture, Medecine and psychiatry, vol. 11.

${ }^{15}$ E. Corin, G. Bibeau, R. Laplante et J.C. Martin (1989). La santé mentale et ses ancrages culture/s. Mineurs, forestiers et agriculteurs de l'Abitibi face à leurs problèmes de santé mentale. Unité de recherche psychosociale, Centre de Recherche de I'Hôpital Douglas.

${ }^{16} \mathrm{M}$. Foucault (1971). Histoire de la folie à l'âge classique. Paris : Union Générale d'édition.

${ }^{17}$ H.F. Ellenberger (1978). Les mouvements de libération mythique et autres essais sur l'histoire de la psychiatrie. Montréal : Éditions Quinze/Critère.

${ }^{18}$ A. Cellar (1989). Folie et société au Québec : de la nouvelle France au milieu du XIXe siècle. Thèse de doctorat en histoire, Université d'Ottawa.

${ }^{19} \mathrm{H}$. Dorvil (1988). De l'Annonciation à Montréal-Histoire de la folie dans la communauté 1962-1987, Montréal : Éditions Émile-Nelligan. Voir aussi du même auteur (1986) : La représentation sociale de la maladie mentale dans une région voisine d'un hôpital psychiatrique du Québec. Thèse de doctorat en sociologie, Université de Montréal.

${ }^{20} \mathrm{E}$. Roosens (1979). Des fous dans la ville ? Gheel et sa thérapie séculaire. Paris : PUF, p. 68.

${ }^{21}$ D. Jodelet (1989). Folies et représentations sociales. Paris : PUF.

${ }^{22}$ Cité dans C. Delacampagne, R. Maggiori (sous la direction de) (1980). Philosopher, les interrogations contemporaines, p. 174. (Matériaux pour un enseignement). Paris : Fayard.

${ }^{23}$ Entretien avec R. Chartier dans É. Vigne (1982). "La naissance de la marginalité ", L'histoire, $n^{\circ} 43:$ 106-111. 
${ }^{24} \mathrm{H}$. Becker (1985). Outsiders-Études de sociologie de la déviance. Paris : Éditions A.M. Métaillé.

${ }^{25} \mathrm{H}$. Collomb, op. cit.

${ }^{26}$ J. Gould et W.K. Kolb (éd.) (1967). A Dictionary of the social sciences. Ouvrage collectif sous les auspices de I'UNESCO. New York: The Free Press, p. 662.

${ }^{27}$ Cité dans Gould et Kolb, op. cit.

${ }^{28}$ Émile Durkheim (1985). Le suicide : étude de sociologie. Paris : Presses Universitaires de France.

${ }^{29}$ W.I. Thomas et S.W. Znaniecki (1919). The Polish Peasant in Europe and America (2 vol.). Chicago : Badger.

${ }^{30}$ R.E.L. Faris (1948). Social Desorganization. New York : Ronald Press.

${ }^{31}$ A.P. Herman (1949). On Approach to Social Problems. Boston : Ginn.

$32 \mathrm{~J}$. Horton (1966). "Order and conflict theories of social problems as competing ideologies ", American Journal of Sociology, vol. 71, $\mathrm{n}^{\circ} 6$.

${ }^{33}$ R. Mayer (1977). Problèmes sociaux (2) : les théories de l'ordre et du conflit en regard des problèmes sociaux (Service social - 2463). Notes de lectures et de cours, École de service social, Université de Montréal.

${ }^{34} \mathrm{H}$. Dorvil (1973). Psychiatrie et antipsychiatrie : un même couple idéologique. Mémoire de maîtrise, École de service social, Université de Montréal.

${ }^{35}$ D. Cooper (1970). Psychiatrie et antipsychiatrie. Paris : Seuil. Voir aussi : R. Laing (1969). Le moi divisé. Paris : Stock. Voir également R. Laing (1969). La politique de l'expérience. Paris : Stock.

${ }^{36}$ De dopamine, le principal neurotransmetteur impliqué dans la psychose. Les médicaments neuroleptiques que l'on prescrit aux psychotiques sont des antidopaminergiques qui agissent à la base des cellules nerveuses pour empêcher les hallucinations et le délire. C'est grâce aux neurotransmetteurs (60 déjà découverts) que le cerveau peut jouer son rôle de recevoir, transmettre et traiter les informations. II faut ajouter que la recherche s'intéresse de plus en plus aux effets secondaires de ces médicaments puissants. 